\title{
Comparison of respiratory muscle strength in individuals performing continuous and noncontinuous walking exercises in water after the 6-week program
}

\author{
Yoshihiro Yamashina 1,* , Hiroki Aoyama', Hirofumi Hori', Emiko Morita', Nami Sakagami', Tomoko Hirayama', Yosuke Yamato', \\ Hiroto Honda', Shigeru Terada', Masahiro Goto' ${ }^{1}$ Kazuyuki Tabira² \\ 'Department of Physical Therapy, Faculty of Health Science, Aino University, Osaka, Japan \\ ${ }^{2}$ Department of Physical Therapy, Faculty of Health Science, Kio University, Nara, Japan
}

This study aimed to compare respiratory muscle strength in individuals performing continuous and noncontinuous walking exercises in water after the 6-week program. Twenty-nine healthy men were randomly divided into a continuous group (CG, $n=14$ ) and a noncontinuous group (NG, $n=15)$. Firstly, both groups executed the 6-week program which set them to walk for 30 min, 4 times per week, over 6 weeks in a pool with the adjusted intensity that their walking speed increased the heart rate to $60 \%$ of the predicted maximum. After the 6-week program, participants in the CG continued the water-walking program for 4 weeks while those in the NG discontinued the water-walking program. In both groups, respiratory muscle strength evaluated by maximum inspiratory pressure (PImax) and maximum expiratory pressure (PEmax) increased significantly after the 6 -week program compared with pre-exercise val- ue. Compared to the value after the 6-week program, PEmax increased significantly in the $C G$ after 10 weeks $(P<0.05)$, but the PImax showed no significant change. Compared to the value after the 6-week program, after 10 weeks, in the NG, PEmax decreased significantly, with a considerable decrease after 9 and 10 weeks compared to the CG $(P<0.05)$. The PImax in the NG significantly decreased after 10 weeks from the value observed after the 6 -week program. We demonstrated that 6 weeks of walking in water at $60 \%$ of the predicted maximum heart rate enhances PImax and PEmax, and that PImax and PEmax decrease in 4 weeks without a water-walking program.

Keywords: Walking in water, Respiratory muscle strength, Healthy males

\section{INTRODUCTION}

As a part of health enhancement plans, aquatic exercise has recently been introduced to rehabilitation and sports clubs (Ide et al., 2005; Jung et al., 2014; Wilcock et al., 2006). Aquatic exercise can reduce the self-weight load, which enables those with obesity (Sheldahl, 1986), joint diseases (Chi et al., 2011), or lumbago (Irandoust et al., 2015) to perform the exercise safely. In addition, it is known that water viscosity and pressure can be used as exercise loads contributing to the enhancement of extremity muscle strength (Craig and Ware, 1967; Ha et al., 2018; Lee et al., 2015).

Breathing underwater requires great effort mainly for the following two reasons: first, the blood volume shifts into the chest cavity because of the increased venous return from the lower extremities; second, inflexibility of the chest wall and diaphragm shifts toward the cranial side because of the hydrostatic pressure, resulting in restricted pulmonary compliance (Craig and Ware, 1967; Craig et al., 1975; de Souto et al., 2012; McNamara et al., 2013). Regarding the effect of the water depth on the respiratory function, the pulmonary vital capacity (VC), forced expiratory volume in $1 \mathrm{sec}\left(\mathrm{FEV}_{1}\right)$, and functional residual capacity decrease during water immersion at the clavicular or cervical level (Agostoni et al., 1966; Buono, 1983; Leith and Mead, 1967; Prefaut et al., 1976). Furthermore, de Andrade et al. (2014) reported that the decrease in the maximum inspiratory muscle strength during water immersion was greater when the water level was at the clav-
${ }^{*}$ Corresponding author: Yoshihiro Yamashina (iD https://orcid.org/0000-0002-0169-5911 Department of Physical Therapy, Faculty of Health Science, Aino University, 4-5-4, Higashioda, Ibaraki City, Osaka 567-0012, Japan

E-mail: y-yamashina@pt-u.aino.ac.jp

Received: May 21, 2019 / Accepted: June 21, 2019
This is an Open Access article distributed under the terms of the Creative Commons Attribution Non-Commercial License (http://creativecommons.org/licenses/by-nc/4.0/) which permits unrestricted non-commercial use, distribution, and reproduction in any medium, provided the original work is properly cited. 
icle than at the xiphoid process because of the higher water pressure at the clavicular level. We also suggested that forced respiration during upright water immersion up to the clavicular level resulted in greater inspiratory muscle fatigue than at shallower depths (Yamashina et al., 2016a).

Therefore, aquatic exercise, such as swimming or walking in water, is ideal for health promotion, which can incorporate resistance training when considering water viscosity during aerobic exercise. In fact, we reported that greater inspiratory and expiratory muscle fatigue was induced by walking in water than walking on land at the same exercise intensity in healthy young men (Yamashina et al., 2016b). In addition, we demonstrated that 6 weeks of walking on land and in water at $60 \%$ of the predicted maximum heart rate enhances respiratory muscle strength (Yamashina et al., 2018).

However, the duration of respiratory muscle strengthening after walking in water has not been studied. Therefore, the purpose of this study was to compare respiratory muscle strength in continuous and noncontinuous groups after walking in water for 6 weeks.

\section{MATERIALS AND METHODS}

\section{Compliance with ethical standards}

The present study was approved by the Institutional Review Board of Aino University (approval number: Aino2016-002). The present study also conformed to the standard set by the Declaration of Helsinki.

\section{Subjects}

We recruited healthy men without exercise habits aged between 20 and 29 years. Subjects with a phobia of water, a history of respiratory or cardiovascular disease, hypertension (resting systolic blood pressure $[\mathrm{BP}] \geq 140 \mathrm{mmHg}$ and/or diastolic $\mathrm{BP} \geq 90$ $\mathrm{mmHg}$ ), diabetes, obesity (body mass index $\geq 30 \mathrm{~kg} / \mathrm{m}^{2}$ ), or a habit of smoking were excluded. Twenty-five eligible applicants met the inclusion criteria and participated in the study.

\section{Informed consent}

Written informed consent was obtained from all subjects prior to the initiation of the present study.

\section{Experimental protocol}

\section{Effects of the 6-week walking in water program on respiratory muscles}

The subjects were randomly divided into two groups, i.e., the
Table 1. The physical characteristics of the participants

\begin{tabular}{lcc}
\hline Characteristic & Continuous group $(\mathrm{n}=14)$ & Noncontinuous group $(\mathrm{n}=15)$ \\
\hline Age $(\mathrm{yr})$ & $26.5 \pm 2.3$ & $25.8 \pm 2.7$ \\
Weight $(\mathrm{kg})$ & $66.8 \pm 6.3$ & $65.9 \pm 5.6$ \\
Height $(\mathrm{cm})$ & $169.4 \pm 5.1$ & $166.5 \pm 4.1$ \\
$\% \mathrm{VC}(\%)$ & $91.4 \pm 6.1$ & $89.6 \pm 7.4$ \\
$\mathrm{FEV}_{1}(\%)$ & $87.1 \pm 3.8$ & $90.7 \pm 5.2$
\end{tabular}

Values are presented as mean \pm standard deviation.

$\% \mathrm{VC}, \%$ vital capacity; $\mathrm{FEV}_{1}$, forced expiratory volume in $1 \mathrm{sec}$.

continuous group (CG, $\mathrm{n}=14$ ) and noncontinuous group (NG, $\mathrm{n}=15$ ) (Table 1). One week before starting the walking program, each individual's weight, height, and respiratory function were measured to confirm that there were no problems with respiratory function.

We used these measured respiratory functions as the pre-exercise values. The maximum inspiratory pressure (PImax) and maximum expiratory pressure (PEmax) in the oral cavity were evaluated using a sthenometer attached to the spirometer (AAM337, Minato, Osaka, Japan) according to the method by Black and Hyatt (1969) and were considered as the surrogate indices of inspiratory and expiratory muscle strength, respectively.

For the walking program, both groups walked in a pool filled to the level of the fourth intercostal space or above. The exercise intensity was adjusted so that the walking speed raised the heart rate to $60 \%$ of the predicted maximum in both groups, and walking continued for 30 min 4 times per week over 6 weeks. A wristtype pulsometer (ForeAthlete 235J, Garmin, Kansas City, MO, USA) was used for the heart rate measurement. Trouser-type swimsuits (without upper parts) and underwater shoes were used for underwater walks. PImax and PEmax were remeasured on land in a sitting position after 6 weeks.

\section{Effects on respiratory muscles with/without the continuation of walking in water}

After the 6-week walking in water program, the CG continued a program another for 4 weeks and the NG was restricted from any exercises except for daily activities. We measured PImax and PEmax weekly for 4 weeks, after the 6-week walking in water program. Using the data gathered after the 6-week walking in water program as baseline values, the following formula was used to calculate the percentage of change in the respiratory muscles.

Rate of change $(\%)=[($ measured value $)-($ baseline value $)] /$ (baseline value) $\times 100$ 
Table 2. Respiratory muscle strength in the continuous and noncontinuous groups pre- and postexercise

\begin{tabular}{lcc}
\hline Variable & Continuous group & Noncontinuous group \\
\hline PImax $\left(\mathrm{cmH}_{2} \mathrm{O}\right)$ & & \\
Pre-exercise & $105.9 \pm 10.1$ & $110.1 \pm 8.1$ \\
After walking in water for 6 weeks & $115.1 \pm 7.6^{*}$ & $117.8 \pm 8.9^{*}$ \\
PEmax $\left(\mathrm{cmH}_{2} \mathrm{O}\right)$ & & \\
Pre-exercise & $107.5 \pm 8.4$ & $103.3 \pm 6.9$ \\
After walking in water for 6 weeks & $116.8 \pm 11.6^{*}$ & $111.3 \pm 9.7^{*}$ \\
\hline
\end{tabular}

Values are presented as mean \pm standard deviation.

PImax, maximal inspiratory pressure; PEmax, maximal expiratory pressure.

${ }^{*} P<0.05$ vs. pre-exercise.

\section{Statistical analysis}

All statistical analyses were performed using Stat View (SAS Institute Inc., Cary, NC, USA), and all values were presented as mean \pm standard deviation. The physical characteristics and the pre-exercise respiratory muscle strength and after the 6-week walking in water program between both groups were performed using the unpaired $t$-test. The pre- and postexercise respiratory muscle strength were compared using the paired $t$-test. The duration of respiratory muscle strengthening after the 6-week walking in water program was examined by two-way (group $\times$ time) analysis of variance with repeated measurements. In cases where significant group and time effects were detected, subsequent post hoc multiple pairwise comparisons (Bonferroni method) were performed. Statistical significance was defined by a value of $P<0.05$.

\section{RESULTS}

\section{Effects of the 6-week walking in water program on respiratory muscles}

In the CG and the NG, PImax and PEmax increased significantly after the 6-week program compared with the pre-exercise value, and no significant difference was observed between the two groups (Table 2).

\section{Effects on respiratory muscles with/without the continuation of walking in water}

Compared to the value after the 6-week program, the $C G$ showed a significant increase in PEmax after 10 weeks $(P<0.05)$, but the PImax did not show any significant change in the CG. Compared to the value after the 6-week program, the NG showed a significant decrease in PEmax after 10 weeks, and also showed a considerable decrease after 9 and 10 weeks when compared to the CG $(P<0.05)$. The PImax in the NG showed a significant decrease af-
Table 3. Comparison of respiratory muscle strength in the continuous and noncontinuous groups after 6-week program

\begin{tabular}{lcccc}
\hline Rate of change (\%) & 7 Weeks & 8 Weeks & 9 Weeks & 10 Weeks \\
\hline PImax & & & & \\
Continuous group & $0.3 \pm 2.0$ & $1.4 \pm 2.5$ & $0.8 \pm 2.2$ & $1.9 \pm 1.8^{\#}$ \\
Noncontinuous group & $-0.9 \pm 1.5$ & $-0.4 \pm 1.3$ & $-1.2 \pm 1.4$ & $-2.6 \pm 0.6^{*}$ \\
PEmax & & & & \\
Continuous group & $0.4 \pm 2.1$ & $1.1 \pm 1.8$ & $2.7 \pm 2.6^{*}$ & $3.6 \pm 2.4^{* * *}$ \\
Noncontinuous group & $0.1 \pm 2.0$ & $-0.1 \pm 3.0$ & $-2.3 \pm 3.8$ & $-3.2 \pm 4.5^{*}$ \\
\hline
\end{tabular}

Values are presented as mean \pm standard deviation.

PImax, maximal inspiratory pressure; PEmax, maximal expiratory pressure.

${ }^{*} P<0.05$ vs. baseline. ${ }^{\#} P<0.05$ vs. noncontinuous group.

ter 10 weeks when compared to the baseline value and the CG (Table 3).

\section{DISCUSSION}

We found that walking in water for 6 weeks at this exercise intensity level was effective to improve inspiratory and expiratory muscle strength and that respiratory strengths decrease in 4 weeks when ceasing walking in water. In general, in the aquatic environment, the hydrostatic pressure on the abdominal wall causes the diaphragm to lift and to pressurize the lungs, and the hydraulic pressure applied to the chest wall causes a lowering of the compliance of the thorax (Bondi et al., 1996; Buono, 1983; Dahlbäck et al., 1978). It is reported that the chest circumference decreased significantly by about $0.8 \mathrm{~cm}$ due to water pressure when it was clavicle-level when compared to before entering the water (Yamashina et al., 2016a). Furthermore, it is reported that the VC and respiratory muscle strength decrease as the water level deepens than the umbilical level (de Andrade et al., 2014). Therefore, it is thought that inspiratory muscle strength increased in the aquatic environment because of the increased load from the water pressure as it enlarges the thorax during inspiration.

PEmax also increased after 6-week program. Previous studies have reported that the abdominal muscular tonus is crucial for stabilizing the trunk to maintain a standing posture while walking in water (Kaneda et al., 2009), and expiratory muscle fatigue was induced by underwater walking more than by land walking (Yamashina et al., 2016b). These reports show that load is added to the abdominal muscle groups when walking in water. Therefore, the abdominal muscles were continually working against water pressure while walking in the water to fix the trunk, which might have strengthened the expiratory muscles after 6-week program. 
Regarding the influence of continuous water walking on respiratory muscle strength, PEmax in the CG showed a significant increase in the 10th week compared to the baseline at the 6th week. When walking in water, it is necessary to work the abdominal muscles, which are expiratory muscles, and to fix the trunk to move forward while resisting water pressure, so it is thought that PEmax increased even after 10 weeks in the CG compared with the value after the 6-week water program. However, PImax in CG showed no significant change. It has been suggested that walking in water for 6 weeks or more can maintain inspiratory muscle strength but may not provide enough load to increase inspiratory muscle strength. On the other hand, in the NG, PImax and PEmax decreased in 4 weeks after the 6-week program. It is suggested that the continuation of exercise is important to maintain and improve respiratory muscle strength.

There are several limitations to this research. We did not measure the tidal volume while walking in water. Since an increased tidal volume is an element of added load on the inspiratory muscles, it is important to measure this in the future. Moreover, in this study, the walking speed was adjusted to $60 \%$ of the predicted maximal heart rate, but whether the same effect can be obtained at other loads should also be investigated in the future.

In summary, we demonstrated that 6 weeks of walking in water at $60 \%$ of the predicted maximum heart rate enhances inspiratory and expiratory muscle strength, and that respiratory strength decreases in 4 weeks after ceasing a water-walking program. Further studies are needed to develop an exercise regimen that can be utilized in the field of sport or clinical settings that makes the most of the characteristics of submersion and aquatic exercise.

\section{CONFLICT OF INTEREST}

No potential conflict of interest relevant to this article was reported.

\section{ACKNOWLEDGMENTS}

This work was supported by a Grant-in-Aid for Scientific Research from the Ministry of Education, Culture, Sports, Science and Technology of Japan.

\section{REFERENCES}

Agostoni E, Gurtner G, Torri G, Rahn H. Respiratory mechanics during submersion and negative-pressure breathing. J Appl Physiol 1966;21:
251-258.

Black LF, Hyatt RE. Maximal respiratory pressures: normal values and relationship to age and sex. Am Rev Respir Dis 1969;99:696-702.

Bondi KR, Young JM, Bennett RM, Bradley ME. Closing volumes in man immersed to the neck in water. J Appl Physiol 1976;40:736-740.

Buono MJ. Effect of central vascular engorgement and immersion on various lung volumes. J Appl Physiol Respir Environ Exerc Physiol 1983; 54:1094-1096.

Chi D, Back Y, Park G, Ju S, Jang HJ. The effect of aquatic exercise on the peak torque and stability of knee joints of elderly women. J Phys Ther Sci 2011;23:871-873.

Craig AB Jr, Dvorak M. Expiratory reserve volume and vital capacity of the lungs during immersion in water. J Appl Physiol 1975;38:5-9.

Craig AB Jr, Ware DE. Effect of immersion in water on vital capacity and residual volume of the lungs. J Appl Physiol 1967;23:423-425.

Dahlbäck GO, Jönsson E, Linér MH. Influence of hydrostatic compression of the chest and intrathoracic blood pooling on static lung mechanics during head-out immersion. Undersea Biomed Res 1978;5:71-85.

de Andrade AD, Júnior JC, Lins de Barros Melo TL, Rattes Lima CS, Brandão DC, de Melo Barcelar J. Influence of different levels of immersion in water on the pulmonary function and respiratory muscle pressure in healthy individuals: observational study. Physiother Res Int 2014;19:140-146.

de Souto Araujo ZT, de Miranda Silva Nogueira PA, Cabral EE, de Paula Dos Santos L, da Silva IS, Ferreira GM. Effectiveness of low-intensity aquatic exercise on COPD: a randomized clinical trial. Respir Med 2012:106:1535-1543.

Ha GC, Yoon JR, Yoo CG, Kang SJ, Ko KJ. Effects of 12-week aquatic exercise on cardiorespiratory fitness, knee isokinetic function, and Western Ontario and McMaster University osteoarthritis index in patients with knee osteoarthritis women. J Exerc Rehabil 2018;14:870-876.

Ide MR, Belini MA, Caromano FA. Effects of an aquatic versus non-aquatic respiratory exercise program on the respiratory muscle strength in healthy aged persons. Clinics (Sao Paulo) 2005;60:151-158.

Irandoust $\mathrm{K}$, Taheri M. The effects of aquatic exercise on body composition and nonspecific low back pain in elderly males. J Phys Ther Sci 2015;27:433-435.

Jung J, Lee J, Chung E, Kim K. The effect of obstacle training in water on static balance of chronic stroke patients. J Phys Ther Sci 2014;26:437440.

Kaneda K, Sato D, Wakabayashi H, Nomura T. EMG activity of hip and trunk muscles during deep-water running. J Electromyogr Kinesiol 2009;19:1064-1070.

Lee DG, Jeong SK, Kim YD. Effects of underwater treadmill walking training on the peak torque of the knee in hemiplegic patients. J Phys Ther 
Sci 2015;27:2871-2873

Leith DE, Mead J. Mechanisms determining residual volume of the lungs in normal subjects. J Appl Physiol 1967;23:221-227.

McNamara RJ, McKeough ZJ, McKenzie DK, Alison JA. Water-based exercise in COPD with physical comorbidities: a randomised controlled trial. Eur Respir J 2013;41:1284-1291.

Prefaut C, Lupi-h E, Anthonisen NR. Human lung mechanics during water immersion. J Appl Physiol 1976;40:320-323.

Sheldahl LM. Special ergometric techniques and weight reduction. Med Sci Sports Exerc 1986;18:25-30.

Wilcock IM, Cronin JB, Hing WA. Physiological response to water immersion: a method for sport recovery? Sports Med 2006;36:747-765.
Yamashina Y, Aoyama H, Hori H, Morita E, Sakagami N, Hirayama T, Yamato Y, Honda H, Terada S, Sugimoto A, Goto M, Tabira K. Effect of walking in water for 6 weeks on respiratory muscle strength. Arch Phys Health Sport Med 2018;1:15-19.

Yamashina Y, Yokoyama H, Naghavi N, Hirasawa Y, Takeda R, Ota A, Imai D, Miyagawa T, Okazaki K. Forced respiration during the deeper water immersion causes the greater inspiratory muscle fatigue in healthy young men. J Phys Ther Sci 2016a;28:412-418.

Yamashina Y, Yokoyama H, Naghavi N, Hirasawa Y, Takeda R, Ota A, Imai D, Miyagawa T, Okazaki K. Treadmill walking in water induces greater respiratory muscle fatigue than treadmill walking on land in healthy young men. J Physiol Sci 2016b;66:257-264. 